\title{
On Lacunary ideal convergence of some sequences
}

Emrah Evren Kara, Mahmut Dastan and Merve Ilkhan

Department of Mathematics, Duzce University, Duzce, Turkey

Received: 30 August 2016, Accepted: 5 October 2016

Published online: 19 March 2017.

\begin{abstract}
In this paper, new classes of lacunary ideal convergent and lacunary ideal bounded sequences combining an infinite matrix and an Orlicz function are defined. Some properties of these spaces are investigated and also some inclusion relations are obtained.
\end{abstract}

Keywords: Invariant means, ideal convergence, Lacunary sequence, Orlicz functions.

\section{Introduction}

The idea of statistical convergence of real sequences was presented by Fast [2] as a generalization of ordinary convergence. It is a practical tool to study the problems related to convergence of numerical sequences by means of the concept of density. Subsequently, Kostyrko [11] extended the set of statistical convergent sequences to ideal convergent sequences by the aid of ideal $\mathscr{I}$ which is a family of subsets of natural numbers $\mathbb{N}$. Since then, ideal convergence has been studied by many researchers including Kostyrko et al [12], Mursaleen and Alotaibi [22], Mursaleen and Mohiuddine [23,24], Mursaleen et al [25], Das [1], Komisarski [10], Lahiri and Das [15], Şahiner et al [28], Gürdal et al $[5,7,6]$, Tripathy and Hazarika [29,30] some of which are on topological spaces and normed spaces.

A family $\mathscr{I}$ of subsets of a non-empty set $X$ is called an ideal on $X$ if for each $A, B \in \mathscr{I}$, we have $A \cup B \in \mathscr{I}$ and for each $A \in \mathscr{I}$ and $B \subseteq A$, we have $B \in \mathscr{I}$. If $X \notin \mathscr{I}$, it is called a non-trivial ideal. A non-trivial ideal is said to be admissible if it contains all finite subsets of $X$. Throughout the study, by $\mathscr{I}$, we mean an admissible ideal on $\mathbb{N}$.

Recall that a sequence $x=\left(x_{k}\right)$ of reel numbers is said to be ideal convergent to a real number $l$ if for every $\varepsilon>0$ the set $\left\{k \in \mathbb{N}:\left|x_{k}-l\right| \geq \varepsilon\right\}$ belongs to the ideal ([11]). A sequence $x=\left(x_{k}\right)$ of real numbers is called ideal bounded if there is a $K>0$ such that $\left\{k \in \mathbb{N}:\left|x_{k}\right|>K\right\} \in \mathscr{I}([12])$.

By $\omega$, we denote the space of all real valued sequences. Let $\Theta=\left(\theta_{r}\right)$ be an increasing sequences of positive integers such that $\theta_{0}=0$ and $h_{r}=\theta_{r}-\theta_{r-1} \rightarrow \infty$ as $r \rightarrow \infty$. Then $\Theta$ is called a lacunary sequence and the intervals $\left(\theta_{r-1}-\theta_{r}\right]$ specified by $\Theta$ is detoned by $\Lambda_{r}$ for all $r \in \mathbb{N}$. Using lacunary sequences, Tripathy et al [31] introduced the space of lacunary ideal convergent sequences

$$
\left\{x=\left(x_{j}\right) \in \omega:\left\{r \in \mathbb{N}: \frac{1}{h_{r}} \sum_{j \in \Lambda_{r}}\left|x_{j}-L\right| \geq \varepsilon\right\} \in \mathscr{I} \text { for every } \varepsilon>0 \text { and some } L\right\}
$$

which is more extensive than lacunary strongly convergent sequence space defined by Freedman et al [3].

A function $M:[0, \infty) \rightarrow[0, \infty)$ is called an Orlicz function if $M$ is continuous, nondecreasing and convex with $M(0)=0$, 
$M(x)>0$ for $x>0$ and $M(x) \rightarrow \infty$ as $x \rightarrow \infty$. By convexity of $M$ and $M(0)=0$, it is obtained that $M(\lambda x) \leq \lambda M(x)$ for all $\lambda \in(0,1)$.

In [14], it is said that $M$ satisfies $\Delta_{2}$-condition for all $x \in[0, \infty)$ if there exists a constant $K>0$ such that $M(2 x) \leq K M(x)$ and it can be easily seen that $K>2$. Also this is equivalent to the satisfaction of condition $M(L x) \leq K L M(x)$, where $L>1$.

By using the idea of Orlicz function, Lindenstrauss and Tzafriri [16] defined Orlicz sequence space

$$
\ell_{M}=\left\{x \in \omega: \sum_{k=1}^{\infty} M\left(\frac{\left|x_{k}\right|}{\rho}\right)<\infty, \text { for some } \rho>0\right\}
$$

which is a Banach space with the norm

$$
\|x\|=\inf \left\{\rho>0: \sum_{k=1}^{\infty} M\left(\frac{\left|x_{k}\right|}{\rho}\right) \leq 1\right\}
$$

In the literature many authors defined various types of ideal convergent sequence spaces by using Orlicz functions, infinite matrices, lacunary sequences. Some of them can be found in $[4,8,9,19,20,21,27]$.

Let $\sigma$ be an injective mapping from the set of the positive integers to itself such that $\sigma^{p}(n) \neq n$ for all positive integers $n$ and $p$, where $\sigma^{p}(n)=\sigma\left(\sigma^{p-1}(n)\right)$. An invariant mean or a $\sigma$-mean is a continuous linear functional defined on the space of all bounded sequences $\ell_{\infty}$ satisfying following conditions for all $x=\left(x_{n}\right) \in \ell_{\infty}$,

1.if $x_{n} \geq 0$ for all $n$, then $\varphi(x) \geq 0$,

2. $\varphi(e)=1$, where $e=(1,1,1, \ldots)$,

3. $\varphi(S x)=\varphi(x)$, where $S x=\left(x_{\sigma(n)}\right)$.

$V_{\sigma}$ denotes the set of bounded sequences all of whose invariant means are equal which is also called as the space of $\sigma$-convergent sequences. In [26], it is defined by

$$
V_{\sigma}=\left\{x \in \ell_{\infty}: \lim _{k} t_{k n}(x)=l, \text { uniformly in } n, l=\sigma-\lim x\right\}
$$

where

$$
t_{k n}(x)=\frac{x_{n}+x_{\sigma^{1}(n)}+\ldots+x_{\sigma^{k}(n)}}{k+1} .
$$

$\sigma$-mean is called a Banach limit if $\sigma$ is the translation mapping $n \rightarrow n+1$. In this case, $V_{\sigma}$ becomes the set of almost convergent sequences which is denoted by $\hat{c}$ and defined in [17] as

$$
\hat{c}=\left\{x \in \ell_{\infty}: \lim _{k} d_{k n}(x) \text { exists uniformly in } n\right\}
$$

where

$$
d_{k n}(x)=\frac{x_{n}+x_{n+1}+\ldots+x_{n+k}}{k+1}
$$

The space of strongly almost convergent sequences was defined by Maddox [18] as follow:

$$
[\hat{c}]=\left\{x \in \ell_{\infty}: \lim _{k} d_{k n}(|x-l e|) \text { exists uniformly in } n \text { for some } l\right\}
$$


Let $p=\left(p_{k}\right)$ be a sequence of positive real numbers such that $0<h=\inf p_{k} \leq p_{k} \leq H=\sup p_{k}<\infty$. For each $k \in \mathbb{N}$ the inequalities

$$
\left|\alpha_{k}+\beta_{k}\right|^{p_{k}} \leq D\left\{\left|\alpha_{k}\right|^{p_{k}}+\left|\beta_{k}\right|^{p_{k}}\right\}
$$

and

$$
|\alpha|^{p_{k}} \leq \max \left\{1,|\alpha|^{H}\right\}
$$

hold, where $\alpha, \alpha_{k}, \beta_{k} \in \mathbb{C}$ and $D=\max \left\{1,2^{H-1}\right\}$.

Let $A=\left(a_{i j}\right)$ be an infinite matrix of complex numbers $a_{i j}$, where $i, j \in \mathbb{N}$. We write $A x=\left(A_{i}(x)\right)$ if $A_{i}(x)=\sum_{j=1}^{\infty} a_{i j} x_{j}$ converges for each $i \in \mathbb{N}$. Throughout the text, by $t_{k n}(A x)$, we mean

$$
t_{k n}(A x)=\frac{A_{n}(x)+A_{\sigma^{1}(n)}(x)+\ldots+A_{\sigma^{k}(n)}(x)}{k+1}
$$

for all $k, n \in \mathbb{N}$.

A sequence space $X$ is called as solid (or normal) if $\left(\gamma_{k} x_{k}\right) \in X$ whenever $\left(x_{k}\right) \in X$ and $\left(\gamma_{k}\right)$ is a sequence of scalars such that $\left|\gamma_{k}\right| \leq 1$ for all $k \in \mathbb{N}$.

Let $X$ be a sequence space and $K=\left\{k_{1}<k_{2}<\ldots\right\} \subseteq \mathbb{N}$. The sequence space $Z_{K}^{X}=\left\{\left(x_{k_{n}}\right) \in \omega:\left(x_{n}\right) \in X\right\}$ is called $K$-step space of $X$.

A canonical preimage of a sequence $\left(x_{k_{n}}\right) \in Z_{K}^{X}$ is a sequence $\left(y_{n}\right) \in \omega$ defined by

$$
y_{n}=\left\{\begin{array}{cc}
x_{n}, & \text { if } n \in \mathbb{N}, \\
0, & \text { otherwise }
\end{array}\right.
$$

A sequence space $X$ is monotone if it contains the canonical preimages of all its step spaces.

Lemma 1. ([13],p.53) If a sequence space $X$ is solid, then $X$ is monotone.

\section{Main results}

Throughout the study, $p=\left(p_{k}\right)$ and $q=\left(q_{k}\right)$ be bounded sequences of positive real numbers. Let $A$ be an infinite matrix and $M$ be an Orlicz function. We define the following spaces:

$$
\begin{gathered}
\mathscr{I}-N_{\theta, \sigma}^{0}(M, A, p)=\left\{x \in \omega: \mathscr{I}-\lim _{r} \frac{1}{h_{r}} \sum_{k \in \Lambda_{r}}\left[M\left(\frac{\left|t_{k n}(A x)\right|}{\rho}\right)\right]^{p_{k}}=0 \text { for all } n \in \mathbb{N} \text { and some } \rho>0\right\}, \\
\mathscr{I}-N_{\theta, \sigma}(M, A, p)=\left\{x \in \omega: \mathscr{I}-\lim _{r} \frac{1}{h_{r}} \sum_{k \in \Lambda_{r}}\left[M\left(\frac{\left|t_{k n}(A x-l e)\right|}{\rho}\right)\right]^{p_{k}}=0 \text { for all } n \in \mathbb{N}, \text { some } \rho>0 \text { and } l \in \mathbb{C}\right\}, \\
\mathscr{I}-N_{\theta, \sigma}^{\infty}(M, A, p)=\left\{x \in \omega: \frac{1}{h_{r}} \sum_{k \in \Lambda_{r}}\left[M\left(\frac{\left|t_{k n}(A x)\right|}{\rho}\right)\right]^{p_{k}} \text { is } \mathscr{I} \text {-bounded for all } n \in \mathbb{N} \text { and some } \rho>0\right\} .
\end{gathered}
$$


If we take $p_{k}=1$ for all $k \in \mathbb{N}$, then the above spaces are denoted by $\mathscr{I}-N_{\theta, \sigma}^{0}(M, A), \mathscr{I}-N_{\theta, \sigma}(M, A), \mathscr{I}-N_{\theta, \sigma}^{\infty}(M, A)$, respectively.

Theorem 1. The spaces $\mathscr{I}-N_{\theta, \sigma}^{0}(M, A, p), \mathscr{I}-N_{\theta, \sigma}(M, A, p), \mathscr{I}-N_{\theta, \sigma}^{\infty}(M, A, p)$ are linear spaces.

Proof. We prove the result for the space $\mathscr{I}-N_{\theta, \sigma}^{0}(M, A, p)$. The proofs for the other spaces follow similarly. Let $x$ and $y$ be any two elements of the space $\mathscr{I}-N_{\theta, \sigma}^{0}(M, A, p)$. Then there exist $\rho_{1}>0$ and $\rho_{2}>0$ such that

$$
\mathscr{I}-\lim _{r} \frac{1}{h_{r}} \sum_{k \in \Lambda_{r}}\left[M\left(\frac{\left|t_{k n}(A x)\right|}{\rho_{1}}\right)\right]^{p_{k}}=0
$$

and

$$
\mathscr{I}-\lim _{r} \frac{1}{h_{r}} \sum_{k \in \Lambda_{r}}\left[M\left(\frac{\left|t_{k n}(A y)\right|}{\rho_{2}}\right)\right]^{p_{k}}=0
$$

That is, for every $\varepsilon>0$, we have that

$$
T_{1}=\left\{r \in \mathbb{N}: \frac{1}{h_{r}} \sum_{k \in \Lambda_{r}}\left[M\left(\frac{\left|t_{k n}(A x)\right|}{\rho_{1}}\right)\right]^{p_{k}} \geq \frac{\varepsilon}{2 D}\right\} \in \mathscr{I}
$$

and

$$
T_{2}=\left\{r \in \mathbb{N}: \frac{1}{h_{r}} \sum_{k \in \Lambda_{r}}\left[M\left(\frac{\left|t_{k n}(A y)\right|}{\rho_{2}}\right)\right]^{p_{k}} \geq \frac{\varepsilon}{2 D}\right\} \in \mathscr{I} .
$$

Since $\mathrm{M}$ is non-decreasing and convex, it follows from inequality 1 that

$$
\begin{aligned}
\frac{1}{h_{r}} \sum_{k \in \Lambda_{r}}\left[M\left(\frac{\left|t_{k n}(A(a x+b y))\right|}{\rho}\right)\right]^{p_{k}} & \leq \frac{1}{h_{r}} \sum_{k \in \Lambda_{r}}\left[M\left(\frac{\left|t_{k n}(A x)\right|}{\rho_{1}}\right)+M\left(\frac{\left|t_{k n}(A y)\right|}{\rho_{2}}\right)\right]^{p_{k}} \\
& \leq D\left\{\frac{1}{h_{r}} \sum_{k \in \Lambda_{r}}\left[M\left(\frac{\left|t_{k n}(A x)\right|}{\rho_{1}}\right)\right]^{p_{k}}+\frac{1}{h_{r}} \sum_{k \in \Lambda_{r}}\left[M\left(\frac{\left|t_{k n}(A y)\right|}{\rho_{2}}\right)\right]^{p_{k}}\right\},
\end{aligned}
$$

where $\rho=\max \left\{2|a| \rho_{1}, 2|b| \rho_{2}\right\}$ and $a, b \in \mathbb{C}$.

If $r \notin T_{1} \cup T_{2}$, then we obtain that

$$
\frac{1}{h_{r}} \sum_{k \in \Lambda_{r}}\left[M\left(\frac{\left|t_{k n}(A(a x+b y))\right|}{\rho}\right)\right]^{p_{k}}<\varepsilon
$$

Therefore the inclusion

$$
\begin{aligned}
\left\{r \in \mathbb{N}: \frac{1}{h_{r}} \sum_{k \in \Lambda_{r}}\left[M\left(\frac{\left|t_{k n}(A(a x+b y))\right|}{\rho}\right)\right]^{p_{k}} \geq \varepsilon\right\} & \subseteq\left\{r \in \mathbb{N}: \frac{1}{h_{r}} \sum_{k \in \Lambda_{r}}\left[M\left(\frac{\left|t_{k n}(A x)\right|}{\rho_{1}}\right)\right]^{p_{k}} \geq \frac{\varepsilon}{2 D}\right\} \\
& \cup\left\{r \in \mathbb{N}: \frac{1}{h_{r}} \sum_{k \in \Lambda_{r}}\left[M\left(\frac{\left|t_{k n}(A y)\right|}{\rho_{2}}\right)\right]^{p_{k}} \geq \frac{\varepsilon}{2 D}\right\}
\end{aligned}
$$

holds. Since the sets in the right side of the inclusion belong to the ideal, this implies that

$$
\left\{r \in \mathbb{N}: \frac{1}{h_{r}} \sum_{k \in \Lambda_{r}}\left[M\left(\frac{\left|t_{k n}(A(a x+b y))\right|}{\rho}\right)\right]^{p_{k}} \geq \varepsilon\right\} \in \mathscr{I}
$$

which means $a x+b y \in \mathscr{I}-N_{\theta, \sigma}^{0}(M, A, p)$. Hence we conclude that $\mathscr{I}-N_{\theta, \sigma}^{0}(M, A, p)$ is a linear space. 
Theorem 2. Let $M_{1}$ and $M_{2}$ Orlicz functions, then we have

$$
\begin{aligned}
& \mathscr{I}-N_{\theta, \sigma}^{0}\left(M_{1}, A, p\right) \cap \mathscr{I}-N_{\theta, \sigma}^{0}\left(M_{2}, A, p\right) \subseteq \mathscr{I}-N_{\theta, \sigma}^{0}\left(M_{1}+M_{2}, A, p\right), \\
& \mathscr{I}-N_{\theta, \sigma}\left(M_{1}, A, p\right) \cap \mathscr{I}-N_{\theta, \sigma}\left(M_{2}, A, p\right) \subseteq \mathscr{I}-N_{\theta, \sigma}\left(M_{1}+M_{2}, A, p\right), \\
& \mathscr{I}-N_{\theta, \sigma}^{\infty}\left(M_{1}, A, p\right) \cap \mathscr{I}-N_{\theta, \sigma}^{\infty}\left(M_{2}, A, p\right) \subseteq \mathscr{I}-N_{\theta, \sigma}^{\infty}\left(M_{1}+M_{2}, A, p\right) .
\end{aligned}
$$

Proof. Let $x \in \mathscr{I}-N_{\theta, \sigma}^{0}\left(M_{1}, A, p\right) \cap \mathscr{I}-N_{\theta, \sigma}^{0}\left(M_{2}, A, p\right)$. Then, given any $\varepsilon>0$ we have

$$
T_{1}=\left\{r \in \mathbb{N}: \frac{1}{h_{r}} \sum_{k \in \Lambda_{r}}\left[M_{1}\left(\frac{\left|t_{k n}(A x)\right|}{\rho}\right)\right]^{p_{k}} \geq \frac{\varepsilon}{2 D}\right\} \in \mathscr{I}
$$

and

$$
T_{2}=\left\{r \in \mathbb{N}: \frac{1}{h_{r}} \sum_{k \in \Lambda_{r}}\left[M_{2}\left(\frac{\left|t_{k n}(A x)\right|}{\rho}\right)\right]^{p_{k}} \geq \frac{\varepsilon}{2 D}\right\} \in \mathscr{I}
$$

for some $\rho>0$. Let $r \notin T_{1} \cup T_{2}$. By the following inequality

$$
\begin{aligned}
& \frac{1}{h_{r}} \sum_{k \in \Lambda_{r}}\left[\left(M_{1}+M_{2}\right)\left(\frac{\mid t_{k n}(A(x) \mid}{\rho}\right)\right]^{p_{k}}=\frac{1}{h_{r}} \sum_{k \in \Lambda_{r}}\left[M_{1}\left(\frac{\mid t_{k n}(A(x) \mid}{\rho}\right)+M_{2}\left(\frac{\mid t_{k n}(A(x) \mid}{\rho}\right)\right]^{p_{k}} \\
& \leq D\left\{\frac{1}{h_{r}} \sum_{k \in \Lambda_{r}}\left[M_{1}\left(\frac{\mid t_{k n}(A(x) \mid}{\rho}\right)\right]^{p_{k}}+\frac{1}{h_{r}} \sum_{k \in \Lambda_{r}}\left[M_{2}\left(\frac{\mid t_{k n}(A(x) \mid}{\rho}\right)\right]^{p_{k}}\right\}
\end{aligned}
$$

and definition of ideal, we obtain that $\left\{r \in \mathbb{N}: \frac{1}{h_{r}} \sum_{k \in \Lambda_{r}}\left[\left(M_{1}+M_{2}\right)\left(\frac{\mid t_{k n}(A(x) \mid}{\rho}\right)\right]^{p_{k}} \geq \varepsilon\right\} \in \mathscr{I}$. This means $x \in \mathscr{I}-N_{\theta, \sigma}^{0}\left(M_{1}+M_{2}, A, p\right)$ and completes the prof. The proof for the other cases follows similarly.

Theorem 3. Let $M_{1}$ and $M_{2}$ be Orlicz functions and $M_{2}$ satisfy $\Delta_{2}$ condition. Then the inclusions

$$
\begin{gathered}
\mathscr{I}-N_{\theta, \sigma}^{0}\left(M_{1}, A, p\right) \subseteq \mathscr{I}-N_{\theta, \sigma}^{0}\left(M_{2} \circ M_{1}, A, p\right), \\
\mathscr{I}-N_{\theta, \sigma}\left(M_{1}, A, p\right) \subseteq \mathscr{I}-N_{\theta, \sigma}\left(M_{2} \circ M_{1}, A, p\right), \\
\mathscr{I}-N_{\theta, \sigma}^{\infty}\left(M_{1}, A, p\right) \subseteq \mathscr{I}-N_{\theta, \sigma}^{\infty}\left(M_{2} \circ M_{1}, A, p\right)
\end{gathered}
$$

hold.

Proof. Let $x \in \mathscr{I}-N_{\theta, \sigma}^{0}\left(M_{1}, A, p\right)$. Then, for some $\rho>0$ we have that $\mathscr{I}-\lim _{r} \frac{1}{h_{r}} \sum_{k \in \Lambda_{r}}\left[M_{1}\left(\frac{\left|t_{k n}(A x)\right|}{\rho}\right)\right]^{p_{k}}=0$. Given any $\varepsilon>0$ choose $\delta$ with $0<\delta<1$ such that $M_{2}(u)<\varepsilon$ whenever $0 \leq u \leq \delta$.

Firstly, assume that $M_{1}\left(\frac{\left|t_{k n}(A x)\right|}{\rho}\right)>\delta$. Since $M_{2}$ satisfies $\Delta_{2}$ condition, there exists $K>1$ such that

$$
M_{2}\left(M_{1}\left(\frac{\left|t_{k n}(A x)\right|}{\rho}\right) \delta^{-1} 2\right) \leq K M_{1}\left(\frac{\left|t_{k n}(A x)\right|}{\rho}\right) \delta^{-1} M_{2}(2)
$$


By using inequalities (2) and (3), we obtain that

$$
\begin{aligned}
\frac{1}{h_{r}} \sum_{k \in \Lambda_{r}}\left[M_{2}\left(M_{1}\left(\frac{\left|t_{k n}(A x)\right|}{\rho}\right)\right)\right]^{p_{k}} & \leq \frac{1}{h_{r}} \sum_{k \in \Lambda_{r}}\left[M_{2}\left(1+M_{1}\left(\frac{\left|t_{k n}(A x)\right|}{\rho}\right) \delta^{-1}\right)\right]^{p_{k}} \\
& \leq \frac{1}{h_{r}} \sum_{k \in \Lambda_{r}}\left[\frac{1}{2} M_{2}(2)+\frac{1}{2} M_{2}\left(M_{1}\left(\frac{\left|t_{k n}(A x)\right|}{\rho}\right) \delta^{-1} 2\right)\right]^{p_{k}} \\
& \leq \frac{1}{h_{r}} \sum_{k \in \Lambda_{r}}\left[\frac{1}{2} M_{2}(2)+\frac{1}{2} K M_{1}\left(\frac{\left|t_{k n}(A x)\right|}{\rho}\right) \delta^{-1} M_{2}(2)\right]^{p_{k}} \\
& \leq \frac{1}{h_{r}} \sum_{k \in \Lambda_{r}}\left[K M_{1}\left(\frac{\left|t_{k n}(A x)\right|}{\rho}\right) \delta^{-1} M_{2}(2)\right]^{p_{k}} \\
& \leq \max \left\{1,\left(K \delta^{-1} M_{2}(2)\right)^{H}\right\} \frac{1}{h_{r}} \sum_{k \in \Lambda_{r}}\left[M_{1}\left(\frac{\left|t_{k n}(A x)\right|}{\rho}\right)\right]^{p_{k}}
\end{aligned}
$$

The following inclusion follows from the last inequality

$\left\{r \in \mathbb{N}: \frac{1}{h_{r}} \sum_{k \in \Lambda_{r}}\left[M_{2}\left(M_{1}\left(\frac{\left|t_{k n}(A x)\right|}{\rho}\right)\right)\right]^{p_{k}} \geq \varepsilon\right\} \subseteq\left\{r \in \mathbb{N}: \frac{1}{h_{r}} \sum_{k \in \Lambda_{r}}\left[M_{1}\left(\frac{\left|t_{k n}(A x)\right|}{\rho}\right)\right]^{p_{k}} \geq \frac{\varepsilon}{\max \left\{1,\left(K \delta^{-1} M_{2}(2)\right)^{H}\right\}}\right\}$.

Hence the set $\left\{k \in \mathbb{N}: \frac{1}{h_{r}} \sum_{k \in \Lambda_{r}}\left[M_{2}\left(M_{1}\left(\frac{\left|t_{k n}(A x)\right|}{\rho}\right)\right)\right]^{p_{k}} \geq \varepsilon\right\}$ belongs to ideal.

Now, suppose that $M_{1}\left(\frac{\mid t_{k n}(A(x) \mid}{\rho}\right) \leq \delta$. Since $M_{2}$ is continuous, for all $\varepsilon>0$ we have $M_{2}\left(M_{1}\left(\frac{\left|t_{k n}(A x)\right|}{\rho}\right)\right)<\varepsilon$. This yields $\frac{1}{h_{r}} \sum_{k \in \Lambda_{r}}\left[M_{2}\left(M_{1}\left(\frac{\left|t_{k n}(A x)\right|}{\rho}\right)\right)\right]^{p_{k}} \leq \max \left\{\varepsilon, \varepsilon^{h}\right\}$. Thus, we conclude that $\mathscr{I}-\lim _{r} \frac{1}{h_{r}} \sum_{k \in \Lambda_{r}}\left[M_{2}\left(M_{1}\left(\frac{\left|t_{k n}(A x)\right|}{\rho}\right)\right)\right]^{p_{k}}=0$ as $\varepsilon \rightarrow 0$ and $r \rightarrow \infty$.

This completes the proof and the other cases can be proved similarly.

Theorem 4. If $\sup _{k}[M(u)]^{p_{k}}<\infty$ for all $u \geq 0$, then the inclusion

$$
\mathscr{I}-N_{\theta, \sigma}(M, A, p) \subseteq \mathscr{I}-N_{\theta, \sigma}^{\infty}(M, A, p)
$$

holds.

Proof. Let $x \in \mathscr{I}-N_{\theta, \sigma}(M, A, p)$. The inequality

$$
\frac{1}{h_{r}} \sum_{k \in \Lambda_{r}}\left[M\left(\frac{\mid t_{k n}(A(x) \mid}{\rho}\right)\right]^{p_{k}} \leq D\left\{\frac{1}{h_{r}} \sum_{k \in \Lambda_{r}}\left[M\left(\frac{\left|t_{k n}(A x-l e)\right|}{\rho_{1}}\right)\right]^{p_{k}}+\frac{1}{h_{r}} \sum_{k \in \Lambda_{r}}\left[M\left(\frac{\left|t_{k n}(l e)\right|}{\rho_{1}}\right)\right]^{p_{k}}\right\}
$$

holds by (1), where $\rho=2 \rho_{1}$. By hypothesis, we have $\frac{1}{h_{r}} \sum_{k \in \Lambda_{r}}\left[M\left(\frac{\left|t_{k n}(l e)\right|}{\rho_{1}}\right)\right]^{p_{k}}<\infty$. Hence, we obtain

$$
\left\{r \in \mathbb{N}: \frac{1}{h_{r}} \sum_{k \in \Lambda_{r}}\left[M\left(\frac{\left|t_{k n}(A x)\right|}{\rho}\right)\right]^{p_{k}} \geq K\right\} \subseteq\left\{r \in \mathbb{N}: \frac{1}{h_{r}} \sum_{k \in \Lambda_{r}}\left[M\left(\frac{\left|t_{k n}(A x-l e)\right|}{\rho_{1}}\right)\right]^{p_{k}} \geq \varepsilon\right\}
$$

for some $K>0$. This completes the proof.

Theorem 5. Let $0<p_{k} \leq q_{k}<\infty$ for every $k \in \mathbb{N}$ and $\left(\frac{q_{k}}{p_{k}}\right)$ be bounded. Then we have

$$
\mathscr{I}-N_{\theta, \sigma}^{0}(M, A, q) \subseteq \mathscr{I}-N_{\theta, \sigma}^{0}(M, A, p)
$$




$$
\mathscr{I}-N_{\theta, \sigma}(M, A, q) \subseteq \mathscr{I}-N_{\theta, \sigma}(M, A, p) .
$$

Proof.It can be easily proved with the same technique as in Theorem 4 in [9].

Theorem 6. The spaces $\mathscr{I}-N_{\theta, \sigma}^{0}(M, A, p)$ and $\mathscr{I}-N_{\theta, \sigma}^{\infty}(M, A, p)$ are solid.

Proof. We prove the result for the space $\mathscr{I}-N_{\theta, \sigma}^{0}(M, A, p)$. For $\mathscr{I}-N_{\theta, \sigma}^{\infty}(M, A, p)$, the result can be proved similarly.

Let $x \in \mathscr{I}-N_{\theta, \sigma}^{0}(M, A, p)$. Then for every $\varepsilon>0$, we have

$$
\left\{k \in \mathbb{N}: \frac{1}{h_{r}} \sum_{k \in \Lambda_{r}}\left[M\left(\frac{\left|t_{k n}(A x)\right|}{\rho}\right)\right]^{p_{k}} \geq \varepsilon\right\} \in \mathscr{I} .
$$

If $\lambda=\left(\lambda_{k}\right)$ is a sequence of scalars such that $\left|\lambda_{k}\right| \leq 1$ for all $k \in \mathbb{N}$, then the following inequality holds:

$$
\frac{1}{h_{r}} \sum_{k \in \Lambda_{r}}\left[M\left(\frac{\left|t_{k n}(A \lambda x)\right|}{\rho}\right)\right]^{p_{k}} \leq \frac{1}{h_{r}} \sum_{k \in \Lambda_{r}}\left[M\left(\frac{\left|t_{k n}(A x)\right|}{\rho}\right)\right]^{p_{k}}
$$

Hence we obtain

$$
\left\{r \in \mathbb{N}: \frac{1}{h_{r}} \sum_{k \in \Lambda_{r}}\left[M\left(\frac{\left|t_{k n}(A \lambda x)\right|}{\rho}\right)\right]^{p_{k}} \geq \varepsilon\right\} \subseteq\left\{k \in \mathbb{N}: \frac{1}{h_{r}} \sum_{k \in \Lambda_{r}}\left[M\left(\frac{\left|t_{k n}(A x)\right|}{\rho}\right)\right]^{p_{k}} \geq \varepsilon\right\}
$$

and so

$$
\left\{r \in \mathbb{N}: \frac{1}{h_{r}} \sum_{k \in \Lambda_{r}}\left[M\left(\frac{\left|t_{k n}(A \lambda x)\right|}{\rho}\right)\right]^{p_{k}} \geq \varepsilon\right\} \in \mathscr{I}
$$

which means $\lambda x \in \mathscr{I}-N_{\theta, \sigma}^{0}(M, A, p)$.

We conclude that the space $\mathscr{I}-N_{\theta, \sigma}^{0}(M, A, p)$ is solid.

Corollary 1. The spaces $\mathscr{I}-N_{\theta, \sigma}^{0}(M, A, p)$ and $\mathscr{I}-N_{\theta, \sigma}^{\infty}\left(M_{1}, A, p\right)$ are monotone.

Proof. The proof follows from Lemma 1.

Theorem 7. If $\lim _{k} p_{k}>0$ and $x \rightarrow l\left(\mathscr{I}-N_{\theta, \sigma}(M, A, p)\right)$, then $l$ is unique.

Proof. Let $\lim _{k} p_{k}=p_{0}>0$. Assume that $x \rightarrow l\left(\mathscr{I}-N_{\theta, \sigma}(M, A, p)\right)$ and $x \rightarrow l^{\prime}\left(\mathscr{I}-N_{\theta, \sigma}(M, A, p)\right)$. Then there exist $\rho_{1}, \rho_{2}>0$ such that

$$
\left\{r \in \mathbb{N}: \frac{1}{h_{r}} \sum_{k \in \Lambda_{r}}\left[M\left(\frac{\left|t_{k n}(A x-l e)\right|}{\rho_{1}}\right)\right]^{p_{k}} \geq \frac{\varepsilon}{2 D}\right\} \in \mathscr{I}
$$

and

$$
\left\{r \in \mathbb{N}: \frac{1}{h_{r}} \sum_{k \in \Lambda_{r}}\left[M\left(\frac{\left|t_{k n}\left(A x-l^{\prime} e\right)\right|}{\rho_{2}}\right)\right]^{p_{k}} \geq \frac{\varepsilon}{2 D}\right\} \in \mathscr{I}
$$

for every $\varepsilon>0$. Put $\rho=\max \left\{2 \rho_{1}, 2 \rho_{2}\right\}$. Hence we have

$$
\frac{1}{h_{r}} \sum_{k \in \Lambda_{r}}\left[M\left(\frac{\left|t_{k n}\left(l e-l^{\prime} e\right)\right|}{\rho}\right)\right]^{p_{k}} \leq D\left\{\frac{1}{h_{r}} \sum_{k \in \Lambda_{r}}\left[M\left(\frac{\left|t_{k n}(A x-l e)\right|}{\rho_{1}}\right)\right]^{p_{k}}+\frac{1}{h_{r}} \sum_{k \in \Lambda_{r}}\left[M\left(\frac{\left|t_{k n}\left(A x-l^{\prime} e\right)\right|}{\rho_{2}}\right)\right]^{p_{k}}\right\} .
$$


This inequality implies that $\quad\left\{r \in \mathbb{N}: \frac{1}{h_{r}} \sum_{k \in \Lambda_{r}}\left[M\left(\frac{\left|t_{k n}\left(l e-l^{\prime} e\right)\right|}{\rho}\right)\right]^{p_{k}} \geq \varepsilon\right\} \quad \mathscr{I}$; that is, $\mathscr{I}-\lim _{r} \frac{1}{h_{r}} \sum_{k \in \Lambda_{r}}\left[M\left(\frac{\left|t_{k n}\left(l e-l^{\prime} e\right)\right|}{\rho}\right)\right]^{p_{k}}=0$. Also we have

$$
\left[M\left(\frac{\left|t_{k n}\left(l e-l^{\prime} e\right)\right|}{\rho}\right)\right]^{p_{k}} \rightarrow\left[M\left(\frac{\left|t_{k n}\left(l e-l^{\prime} e\right)\right|}{\rho}\right)\right]^{p_{0}}
$$

as $k \rightarrow \infty$ and so $\left[M\left(\frac{\left|t_{k n}\left(l e-l^{\prime} e\right)\right|}{\rho}\right)\right]^{p_{0}}=0$. Hence, we conclude that $l=l^{\prime}$.

\section{Acknowledgments}

The authors would like to thank the anonymous referee for his/her comments that helped us improve this article.

\section{Competing interests}

The authors declare that they have no competing interests.

\section{Authors' contributions}

All authors have contributed to all parts of the article. All authors read and approved the final manuscript.

\section{References}

[1] P. Das, Some further results on ideal convergence in topological spaces, Topology Appl. 159(10-11) (2012) 2621-2626.

[2] H. Fast, Sur la convergence statistique, Colloq. Math. 2(3-4) (1951) 241-244.

[3] A. R. Freedman, J. J. Sember and M. Raphael: Some Cesaro-type summability spaces, Proc. London Math. Soc. 37(3) (1978) 508-520.

[4] B. Hazarika, Strongly almost ideal convergent sequence spaces in a locally convex space defined by Musielak-Orlicz function, Iran. J. Math. Sci. Inform. 9(2) (2014) 15-35.

[5] M. Gürdal, On ideal convergent sequences in 2-normed spaces, Thai J. Math. 4(1) (2006) 85-91.

[6] M. Gürdal and M. B. Huban, On I-convergence of double sequences in the Topology induced by random 2-norms, Mat. Vesnik 66(1) (2014) 73-83.

[7] M. Gürdal and A. Şahiner, Ideal Convergence in n-normed spaces and some new sequence spaces via n-norm, J. Fundamental Sci. 4(1) (2008) 233-244.

[8] E. E. Kara and M. İlkhan, On some paranormed A-ideal convergent sequence spaces defined by Orlicz function, Asian J. Math. Comput. Research 4(4) (2015) 183-194.

[9] E. E. Kara and M. İlkhan, Lacunary $\mathscr{I}$-convergent and lacunary $\mathscr{I}$-bounded sequence spaces defined by an Orlicz function, Electron. J. Math. Anal. Appl. 4(2) (2016) 150-159.

[10] A. Komisarski, Pointwise I-convergence and I-*convergence in measure of sequences of functions, J. Math. Anal. Appl. 340(2) (2008) 770-779.

[11] P. Kostyrko, W. Wilczynski and T. Salat, $\mathscr{I}$-convergence, Real Anal. Exchange 26(2) (2000) 669-686.

[12] P. Kostyrko, M. Macaj, T. Salat and M. Sleziak, I-convergence and external I-limit points, Math. Slovaca 55(4) (2005) $443-464$.

[13] P. K. Kamptan and M. Gupta, Sequence spaces and series, Marcel Dekker, New York, 1980.

[14] M. A. Krasnoselskii and Y. B. Rutitcky, Convex functions and Orlicz spaces, P.Noordhoff, Groningen, The Netherlands, 1961.

[15] B. K. Lahiri and P. Das, I and I*-convergence in topological spaces, Math. Bohem. 130(2) (2005) 153-160.

[16] J. Lindenstrauss and L. Tzafriri,On Orlicz sequence spaces, Israel J. Math. 10(3) (1971) 379-390. 
[17] G. G. Lorentz, A contribution to the theory of divergent series, Act. Math. 80 (1948) 167-190.

[18] I. J. Maddox, Spaces of strongly summable sequences, Q. J. Math. 18 (1967) 345-355.

[19] S. A. Mohiuddine and M. Aiyub, Lacunary statistical convergence in random 2-normed spaces, Appl. Math. Inf. Sci. 6(3) (2012) 581-585.

[20] S. A. Mohiuddine and M. A. Alghamdi, Statistical summability through a lacunary sequence in locally solid Riesz spaces, J. Inequal. Appl. 2012 (2012) Article ID 225.

[21] M. Mursaleen and S. A. Mohiuddine, On lacunary statistical convergence with respect to the intuitionistic fuzzy normed space, J. Comput. Appl. Math. 233(2) (2009) 142-149.

[22] M. Mursaleen and A. Alotaibi, On I-convergence in random 2-normed spaces, Math. Slovaca 61(6) (2011) 933-940.

[23] M. Mursaleen and S. A. Mohiuddine, On ideal convergence of double sequences in probabilistic normed spaces, Math. Reports 12(64)(4) (2010) 359-371.

[24] M. Mursaleen and S. A. Mohiuddine, On ideal convergence in probabilistic normed spaces, Math. Slovaca 62 (2012) 49-62.

[25] M. Mursaleen, S. A. Mohiuddine and O. H. H. Edely, On the ideal convergence of double sequences in intuitionistic fuzzy normed spaces, Comput. Math. Appl. 59 (2010) 603-611.

[26] P. Schaefer, Infinite matrices and invariant means, Proc. Amer. Math. Soc. 36 (1972) 104-110.

[27] A. Şahiner,On I-lacunary strong convergence in 2-normed spaces, Int. J. Contempt. Math. Sciences 2(20) (2007) 991-998.

[28] A. Şahiner, M. Gürdal, S. Saltan and H. Gunawan, Ideal convergence in 2-normed spaces, Taiwanese J. Math. 11(5) (2007) 14771484.

[29] B. C. Tripathy and B. Hazarika, Paranorm I-convergent sequence spaces, Math. Slovaca 59(4) (2009) 485-494.

[30] B. C. Tripathy and B. Hazarika, I-monotonic and $I$-convergent sequences, Kyungpook Math. J. 51 (2011) $233-239$.

[31] B. C. Tripathy, B. Hazarika and B. Choudhary, Lacunary $\mathscr{I}$-convergent sequences, Kyungpook Math. J. 52 (2012) $473-482$. 